\title{
Batch fabricated sensors for magnetic force microscopy
}

\author{
P. Grütter, D. Rugar, H. J. Mamin, G. Castillo, S. E. Lambert, C.-J. Lin, \\ and R. M. Valietta \\ IBM Research Division, Almaden Research Center, 650 Harry Road, San Jose, California $95120-6099$
}

O. Wolter, T. Bayer, and J. Greschner

IBM Deutschland GmbH, GMTC, 7032 Sindelfingen, West Germany

\section{(Received 20 July 1990; accepted for publication 20 August 1990)}

\begin{abstract}
In this letter we describe force sensors suitable for magnetic force microscopy (MFM) made by coating silicon microcantilevers with various thin magnetic films. These MFM force sensors can be batch fabricated and their magnetic propertics tailored by choosing appropriate coatings. This is demonstrated by analyzing the MFM response for magnetization patterns written on a longitudinal recording test sample. Selectable components of the sample stray fidd can be measured by coating the tips with high coercivity films. Tips coated with low coercivity films such as Permalloy respond to the total stray field. Sample perturbations due to the tip stray field are small for thin-film tips, allowing the imaging of low coercivity samples such as Permalloy.
\end{abstract}

Magnetic force microscopy ${ }^{1,2}$ (MFM) is a rapidly evolving technique for imaging magnetic microstructure with high resolution and minimal sample preparation. The crucial component of every MFM is the force sensor (a sharp magnetic tip on a soft cantilever). Previously, force sensors have been made from fine ferromagnetic wires ${ }^{1,3,4}$ or thin foils ${ }^{5}$ and required individual etching, bending, and handing as described in detail in $\mathbb{R e f}$. 4 . Some groups have successfully coated etched tungsten wires with magnetic thin films. ${ }^{4,6,7}$ In this letter, we demonstrate that it is possible to batch fabricate silicon microcantilevers with magnetic coatings suitable for MPM.

We have developed microcantilevers with integrated sharp, high aspect ratio silicon tips suitable for general purpose force microscopy. ${ }^{8}$ Figure 1 shows the geometry of the force sensors used in this study. The cantilevers are typically $500 \mu \mathrm{m}$ long, $11.5 \mu \mathrm{m}$ wide, and $5-7 \mu \mathrm{m}$ thick. This gives spring constants on the order of $1 \mathrm{~N} / \mathrm{m}$ and resonant frequencies of typically $35 \mathrm{kHz}$. The integrated conical tips are about $10 \mu \mathrm{m}$ long and have a typical cone angle of $10^{\circ}$ or less. This small cone angle is desirable for MFM tips due to the long-ranged nature of the magnetic interaction. The fabrication process for these force sensors differs from those reported previously by Albrecht et al. ${ }^{9}$ in that they are etched from single-crystal silicon. The process, which involves a combination of wet and dry etching techniques, results in tips with both a narrow cone angle and small tip radius (typically $5-10 \mathrm{~nm}$ ).

The force sensors are made magnetically active by coating them with a thin ferromagnetic film. Three types of coatings were evaluated: evaporated $\mathrm{Co}$, sputter-deposited $\mathrm{CO}_{71} \mathrm{PA}_{12} \mathrm{Cr}_{17}$ and sputter-deposited $\mathrm{Ni}_{80} F_{20}$ (Permalloy). These films have magnetization values $M_{s}$ of 1422,450 , and $835 \mathrm{emu} / \mathrm{cm}^{3}$ and coercitivities $H_{c}$ of 190,550 , and 2 Oe, respectively. These film properties were measured on flat silicon coupon samples. The infuence of the sharp tip geometry on magnetic properties is not clear; higher values of coercivity are expected. All films were nominally $15 \mathrm{~nm}$ thick. The magnetic signal obtained with the two high co- ercivity tips was found to be significantly enhanced by magnetizing them in a strong feld of about $10 \mathrm{kG}$.

The instrument used in this study, previously described by Rugar et al., ${ }^{10}$ utilizes an all-iber interferometer to sense the motion of the cantilever. Two modes of attractive force imaging were employed. The first is the standard amplitude detection mode, where shifts of the cantilever resonance curve due to interaction force gradients lead to a measurable reduction of the cantilever oscillation amplitude. ${ }^{11}$ We have also operated the instrument in moderate vacuum $\left(10^{-3}\right.$ Torr), which greatly increases the quality factor $Q$ of the cantilever to more than 20000 (compared to about 50 when operated in air). This higher $Q$ value allows an increase in force gradient sensitivity of at least an order of magniude. However, to image with reasonable bandwidth at this sensitivity, a new detection mode, based on directly measuring the cantilever resonant frequency with an FM detector, was implemented. ${ }^{12}$

We characterized the magnetic properties of the tips by imaging transitions written at diferent frequencies on a test sample consisting of a piece of a longitudinal magnetic recording disk. Tracks $8 \mu \mathrm{m}$ wide with transitions separated by 10 and $2 \mu \mathrm{m}$ were imaged with all tips for direct comparisons. The dominant tip magnetization component can be deduced from the symmetry of the measured signal, as discussed previously by Mamin et al. ${ }^{13}$ We find that both the $\mathrm{Co}$ and the CoPtCr coatings are magnetically hard, in contrast to the NiFe tips, which are magnetically soft.

Figure $2(a)$ is a typical image acquired with a Co-

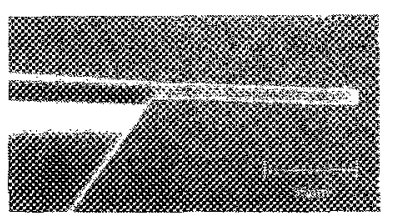

(a)

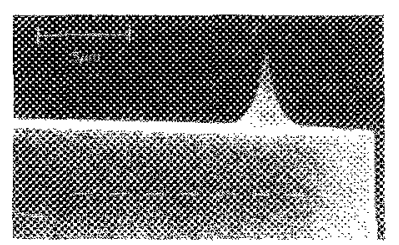

(b)
HIG. 1. (a) Scanning electron microscopy image of microfabricated $\mathrm{Si}$ force sensor with integrated tip; (b) Magnified view of the tip. 


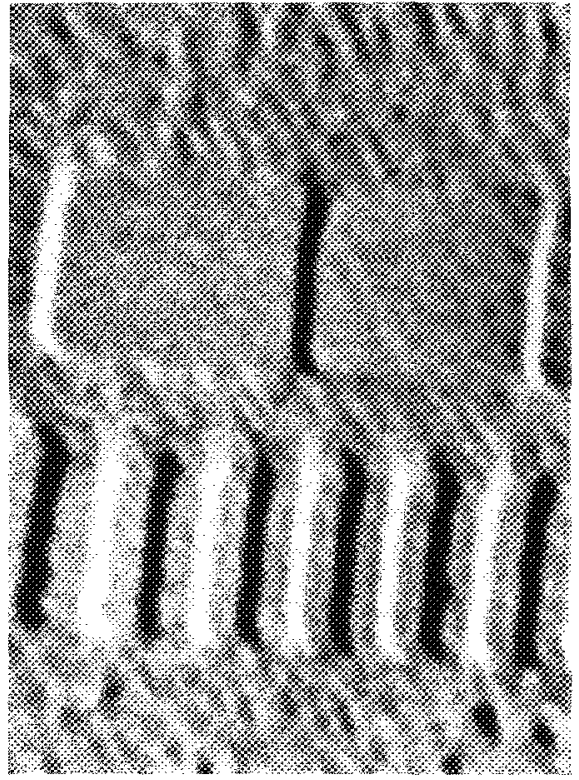

(a)

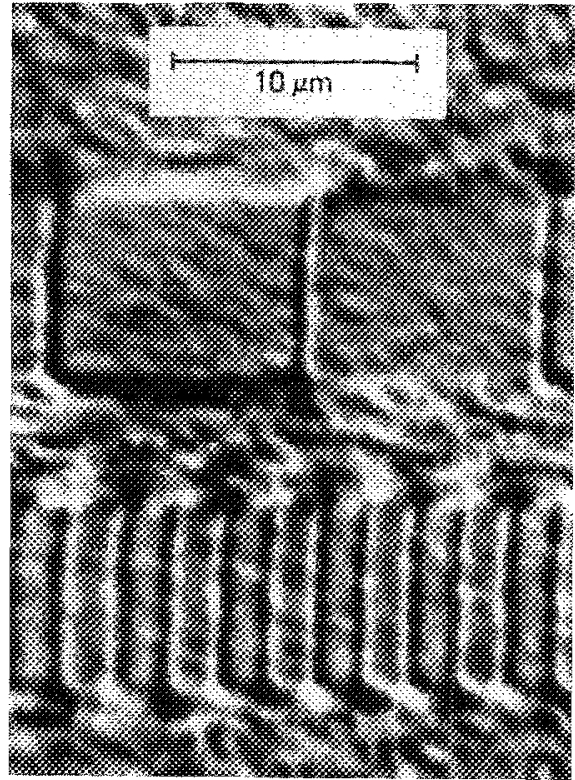

(b)

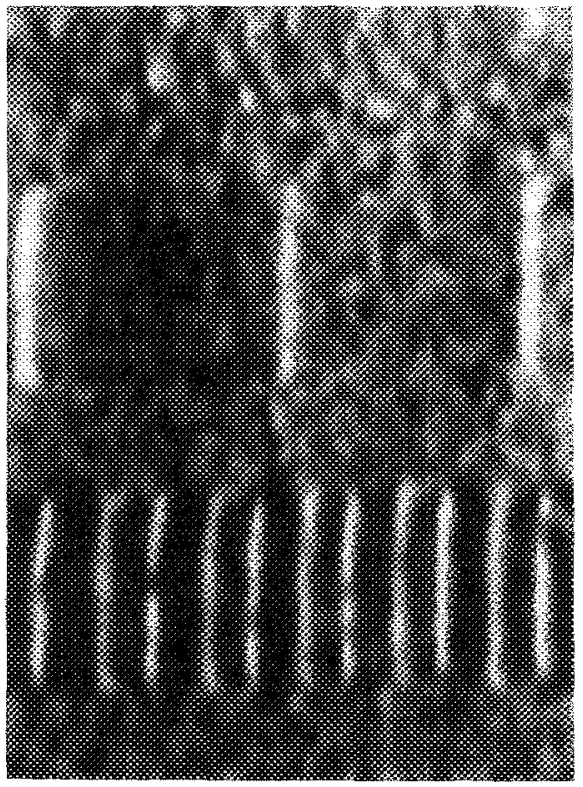

(c)

FIG. 2. MFM images of $8 \cdot \mu \mathrm{m}$-wide tracks on a longitudinal magnetic recording sample. (a) Image acquired with an evaporated Co thin-film tip magnetized perpendicular to the sample. (b) Image acquired with a sputtered CoPtCr tip magnetized parallel to the sample, along the track direction. (c) Image acquired with a sputtered NiFe thiri-film tip. Note that all transitions appear bright (attractive).

coated tip magnetized perpendicular to the sample plane (along the tip axis). It was obtained at a tip-sample spacing of $z \approx 300 \mathrm{~nm}$. The dark and bright transitions are due to at ractive and repulsive magnetic interaction, respectively. Single line scans shown in Fig. 3(a) show these two types of transitions in greater detail. The symmetry of the MFM response above transitions is even [Fig. 3(a)], which is the expected response when the effective tip magnetic moment is oriented perpendicular to the sample plane. ${ }^{4,13,14}$ This axial orientation of the tip magnetization was also found by direct Lorentz microscopy characterization of the tip.

The above results indicate that tips coated with high coercivity films are magnetically hard. We have further demonstrated this by magnetizing a $\mathrm{CoPtCr}$ tip perpendicular to its axis (and thus parallel to the sample plane) in a $15 \mathrm{kG}$ external field to prove that the demagnetization field and the shape anisotropy of the tip are not strong enough to overcome the large coercivity of the $\mathrm{CoPtCr}$ film. Figure

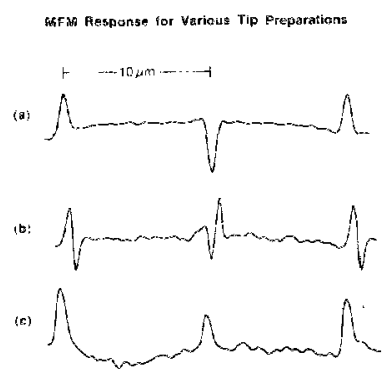

FIG. 3. Averaged scanlines corrcsponding to the $10 \mu \mathrm{m}$ transitions in Fig. 2. The even or odd symmetry above a transition is evident for tips magnetized either (a) perpendicular or (b) paralel to the sample, respectively. In (c) the overall attractive response of the NiFe tip leads to maxima above every transition. 2(b) shows an image acquired with this tip at a distance of $100 \mathrm{~nm}$. Each transition results in a pair of bright and dark stripes. The line scans in Fig. 3(b) ciearly show this bipolar response and demonstrate the odd symmetry about a transition. This odd symmetry is expected for a tip magnetic moment oriented predominantly parallel to the sample plane ( $x$ direction) and thus responsive to the in-plane component $\partial^{2} H_{x} / \partial z^{2}$ of the sample stray field. ${ }^{4,13-15}$

Images obiained with magnetically hard tips clearly show repulsive (biack) and attractive (white) interaction over transitions, as expected from the two different types of transitions (head-to-head or tail-to-tail sample magnetization). The behavior of low coercivity NiFe tips is different; all transitions appear to be attractive (white), as shown in Fig. 2(c). This can be understood if the NiFe thin film remains, as might be expected, magnetically soft even upon sputter deposition onto sharp silicon tips. The effective tip magnetic moment orients itself in the stray field emanating from the sample, always leading to an attractive interaction.

Some differences between transition strengths can be seen in Fig. 3(c). In addition, it was observed that the distance dependence of the measured signal was not the same above all transitions. Every other transition became very weak when the tip sample distance exceeded a rather sharp threshold. This threshold distance was largest for transitions written at a low spatial frequency. This behavior can qualitatively be understood as a consequence of the transition stray field distance dependence, which is a function of transition frequency. ${ }^{16}$ In order for the overall magnetic interaction to be attractive, the sample stray field has to be larger than the coercivity of the tip. From a simple 
calculation of fields above transitions we estimate an effective coercivity of about $40 \mathrm{Oe}$ for the thin NiFe film.

In general, high quality images showing fine details can be taken with all of the coated tips. Such images show "magnetic roughness" in the regions outside the tracks, as observable in Fig. 2. This is a refection of the complicated field patterns arising from the demagnetized state of the as-deposited media, which was not erased or magnetized. The oppositely magnetized regions between the transitions do not exhibit this structure, as they were saturated by the head field during the writing process.

The magnetic signal from the evaporated Co force sensors was quite weak; thus satisfactory images could only be acquired with the enhanced sensitivity achievable with the FM detector in vacuum. This is in contrast to measurements with sputter-deposited CoPtCr or NiFe tips, with which images of excellent contrast were obtainable even in air, despite their smaller values of $M_{s}$. This small signal may be due to the lower coercivity of the Co film (leading to lower remanent magnetization) or due to the reduced film thickness along the tip sidewall as a result of shadowing effects during Co evaporation. No evident differences in resolution were observed for tips coated by the diferent deposition techniques. A routine lateral resolution of 50 $\mathrm{nm}$ was obtained with most tips.

Another advantage of these coated tips is the smaller tip stray field compared to a ferromagnetic wire tip. Calculations for a conical $\mathrm{CoPtCr}$ shell $15 \mathrm{~nm}$ thick and 10 $\mu \mathrm{m}$ long with a cone angle of $10^{\circ}$ give a peak stray feld of 3 Oe with a full width at half maximum (FWHM) of 450 $\mathrm{nm}$ at a distance of $100 \mathrm{~nm}$. This has to be compared to the corresponding results for a solid conical wire of identical material and geometry: 68 Oe and $2200 \mathrm{~nm} \mathrm{FWHM}$. The tip stray field from a coated thin-film tip is much smaller and more localized than that of the corresponding wire tip. This is a consequence of the reduced volume of magnetio material in the tip. The reduction in stray field is important when imaging soft magnetic materials, where a large tip stray field can induce domain wall motion in the sample. ${ }^{17,18}$ Furthermore, preliminary calculations indicate that the resulting reduction in imaging sensitivity (force gradient) is not severe.

Experimentally we demonstrate this advantage of thinfilm tips by imaging a $30 \mathrm{~nm}$ thin patterned NiFe sample previously used by Mamin et al. ${ }^{17}$ Figure 4 is an image of a $20 \mu \mathrm{m} \mathrm{NiFe} \mathrm{square,} \mathrm{measured} \mathrm{with} \mathrm{a} \mathrm{CoPtCr} \mathrm{tip} \mathrm{mag-}$ netized along the tip axis. In this initial experiment no tip induced domain wall motion was observed, a result indicative of the expected smaller tip stray field.

In summary, we have demonstrated that it is possible to batch fabricate high sensitivity MFM force sensors with controlled magnetic properties by coating microfabricated Si force sensors with various magnetic thin films. These advances in force sensor technology will make MFM a more practical and easier to use technique with a larger scope of applications. From the measurements performed on a magnetic recording test sample it can be concluded that diferent components of the sampie stray field can be selectively measured by appropriately magnetizing hard

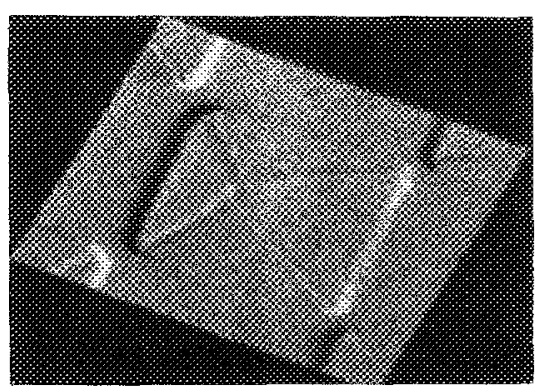

FIG. 4. MFM image of a $20 \mu \mathrm{m}, 30-\mathrm{nm}$-thick NiFe square measured with a spurtered $\mathrm{CoP}^{\mathrm{P}} \mathrm{tCr}$ tip magnetized perpendicular to the sample. The domain walls, including some small bifurcations near the corners, are clearly seen. The image was processed by a light source technique to highlight the domain walls.

magnetic tips in an external field. Intrinsically soft magnetic films, such as NiFe, remain soft even when sputtered onto force sensor tips. Tips coated with thin magnetic films have a significantly reduced perturbation effect on soft magnetic samples (compared to buik wire tips) due to their reduced magnetic volume.

The authors thank T. R. Albrecht, P. Wimmer, and P. Gitthner for assistance in the design and construction of the instrument, R. E. Fontana $\mathbb{J}$. for providing us with the $\mathrm{NiFe}$ sample, I. A. Beardsley for helpful discussions, and I. $R$. McFadyen for the characterization of tips by Lorentz microscopy.

'Y. Martin and H. K. Wickramasinghc, Appl. Phys. Lett. 50, 1455 (1987).

2J. J. Saenz, N. Garcia, P.Grütter, F. Meyer, H. Heinzelmann, R. Wiesendanger, L. Rosenthaler, H. R. Hidber, and II. J. Guntherodt, J. Appl. Phys. 62, 4293 (1987).

${ }^{3} \mathrm{C}$. Schönenberger, Ph.D. thesis, ETH, Zurich, Switzerland 1990.

${ }^{4}$ D. Rugar, I. L. Mamin, P. Guethrer, S. E. Iambert, J. E. Stern, I. McFadyen, and T. Yogi, J. Appl. Phys. 68, 1169 (1990).

'P. Grüter, Ph.D. thesis, University of Basel, 1989.

${ }^{5}$ H. J. Mamin, D. Rugar, P. Grüter, $\mathbb{P}$. Guethner, S. E. Lambert, T. Yogi, O. Wolter, and J. Greschner, Bull. Am. Phys. Soc. 35, 420 (1990).

${ }^{9}$ A. J. den Boef, Appl. Phys. Lett. 56, 2045 (1990).

${ }^{8} \mathrm{O}$. Wolter, Th. Bayer, and J. Greschner, J. Vac. Sci. Technol. A (to be published).

${ }^{9}$ T. R. Albrecht, S. Akamine, T. E. Carver, and C. F. Quate, J. Vac. Sci. Technol. A 8, 3386 (1990).

${ }^{10}$ D. Rugar, H. J. Mamin, and P. Quethner, Appl. Phys. Lett. 55, 2588 (1989).

"Y. Martin, C. C. Williams, and I. K. Wickramasinghe, J. Appl, Phys. 61, 4723 (1987).

${ }^{12}$ T. R. Albrecht, P. Grütter, D. Horne, and D. Rugar, J. Appl. Phys (to be published, 1991).

H. J. Mamin, D. Rugar, J. E. Stern, B. D. Terris, and S. E. Lambert, Appl. Phys. Lett. 53, 1563 (1988).

${ }^{14}$ A. Wadas, E. Grutter, and H. J. Gütherodt, J. Vac. Sci. Technol. A 8, $416(1990)$.

${ }^{15}$ D. W. Abraham, C. C. Wiliams, and H. K. Wickramasinghe, Appl. Phys. Lett. 53, 1446 (1988).

${ }^{16}$ R. L. Wallace, Bell Syst. Technol. J. 30, 145 (1951).

${ }^{17}$ H. J. Mamin, D. Rugar, J. E. Stern, R. E. Fontana, Jr., and Y. Kasiraj, Appl. Phys. Lett. 55, 318 (1989).

${ }^{18} \mathrm{~T}$. Göddenhenrich, U. Hartmann, M. Anders, and C. Heiden, J. Microse. 152, 527 (1988). 